\title{
The quiet Sun's magnetic flux estimated from Ca II H bright inter-granular G-band structures
}

\author{
B. Bovelet and E. Wiehr \\ Institut für Astrophysik, Universität Göttingen, Friedrich-Hund-Platz 1, 37077 Göttingen, Germany \\ e-mail: ewiehr@astro.physik.uni-goettingen.de
}

Received 4 March 2008 / Accepted 5 June 2008

ABSTRACT

\begin{abstract}
Aims. We determine the number density and area contribution of small-scale inter-granular Ca II bright $G$-band structures in images of the quiet Sun as tracers of kilo-Gauss magnetic flux-concentrations.

Methods. In a $149^{\prime \prime} \times 117^{\prime \prime} G$-band image of the disk center at the activity minimum, 7593 small inter-granular structures were segmented with the "multiple-level tracking" pattern recognition algorithm. The scatterplot of the continuum versus the $G$-band brightness shows the known magnetic and non-magnetic branches. These branches are largely disentangled by applying an intrinsic $\mathrm{Ca}$ II H excess criterion. The thus obtained 2995 structures contain $1152 \mathrm{G}$-band bright points (BP) and $1843 G$-band faint points (FP). They show a tendency toward increasing size with decreasing $G$-band excess, as expected from the "hot wall" picture. Their $\mathrm{Ca}$ II H and $G$-band brightness are slightly related, resembling the known relation of Ca II and magnetic field strength. The magnetic flux density of each individual $\mathrm{BP}$ and $\mathrm{FP}$ is estimated from their $G$-band brightness according to MHD model calculations.

Results. The entity of BP and FP covers the total FOV with a number density of $0.32 / \mathrm{Mm}^{2}$ and a total area contribution of $2.0 \%$. Their individual calibrations yield a mean flux density of $20 \mathrm{Mx} / \mathrm{cm}^{2}$ in the entire FOV and $13 \mathrm{Mx} / \mathrm{cm}^{2}$ for inter-network regions.
\end{abstract}

Key words. Sun: magnetic fields - Sun: activity - Sun: photosphere

\section{Introduction}

The small-scale magnetic flux-concentrations on the non-active solar surface are an important ingredient for understanding solar magnetism. Active region features, such as sunspots, faculae, prominences, and flares, exhibit a strong variation with the solar cycle, commonly explained by a global solar dynamo acting in the deep convection zone. On the other hand, small-scale magnetic flux-concentrations exist that cover the solar surface more homogeneously in time and space indicating a less tight dependence on the global dynamo

Unfortunately, it is difficult to measure magnetic flux on those scales predicted by MHD modeling (Vögler \& Schüssler 2007). Measurements of circular polarization due to the Zeeman effect can only give the net flux, since opposite polarities cancel each other out within the spatial resolution element (Domínguez Cerdeña et al. 2006). The absolute (unsigned) flux, however, has to be deduced from magnetically broadened intensity profiles (Stokes- $I$ ) that are affected not only by the magnetic field but also by the, a priori unknown, intrinsic atmosphere of each individual flux concentration.

The problems for such a conversion have been discussed by, e.g., Sánchez Almeida (2003). Algorithms used for this procedure are often called "inversion codes", which might suggest that the observed polarization profiles are actually "inverted" into magnetic field parameters. Instead, these codes vary both the magnetic field structure and the intrinsic atmosphere model until an optimal parameter fit between calculated and observed profiles is found. The codes also optimize the filling factor of the magnetic signal inside the resolution element but do not consider two-dimensional radiative effects in very small structures.

Recent observations impressively show that fluxconcentrations can be of such a small size that even the $0.09^{\prime \prime}$ spatial resolution of the $1 \mathrm{~m} \mathrm{SST}$ at La Palma (Scharmer et al 2003) does not reach the lower end of the size distribution of $G$-band structures. Stokes images, however, cannot achieve such a high spatial resolution, due to additional optical elements and longer exposure times. Hence, they even depend more on the (doubtful) fill factor than intensity images.

Many of the problems in interpreting polarization measurements may be avoided by analyzing intensity images (Sanchez Almeida et al. 2004; De Wijn et al. 2005). Small-scale magnetic concentrations of kilo-Gauss flux density are known to exhibit excess brightness in the $C H$-band near $430 \mathrm{~nm}(G$-band) over the neighboring continuum (Berger et al. 1995), thus allowing their study in post factum reconstructed images of higher spatial and temporal resolution than achieved for Stokes measurements. The excess in the $G$-band brightness over the neighboring spectral continuum is a different measure than the excess over the mean photospheric $G$-band level, defining $G$-band bright points (BP; Muller \& Roudier 1984). The latter cover two families of structures: (I) inter-granular BP, which are magnetic (Berger \& Title 2001) and down-drafting (Langhans et al. 2002), and (II) nonmagnetic BP, which move upwards with the respective granules (same two papers).

We separate both types of BP using a pattern recognition code, (Bovelet \& Wiehr 2007) especially adapted to detect the inter-granular BP. We then select magnetic inter-granular structures using their $G$-band-to-continuum ratio and additionally their Ca II H excess brightness. There is some controversy about these criteria: Rezaei et al. (2007) find that the Ca II excess cannot be considered a general indicator for magnetic flux concentrations. Berger et al. (1998) argue that the $G$-band brightness is not a sufficient criterion for the magnetic nature of a BP. De Wijn et al. (2005) suggest the Ca II brightness to be a more realistic criterion. Their concern about the $G$-band criterion refers to Berger \& Title (2001), who, however, only exclude that criterion 


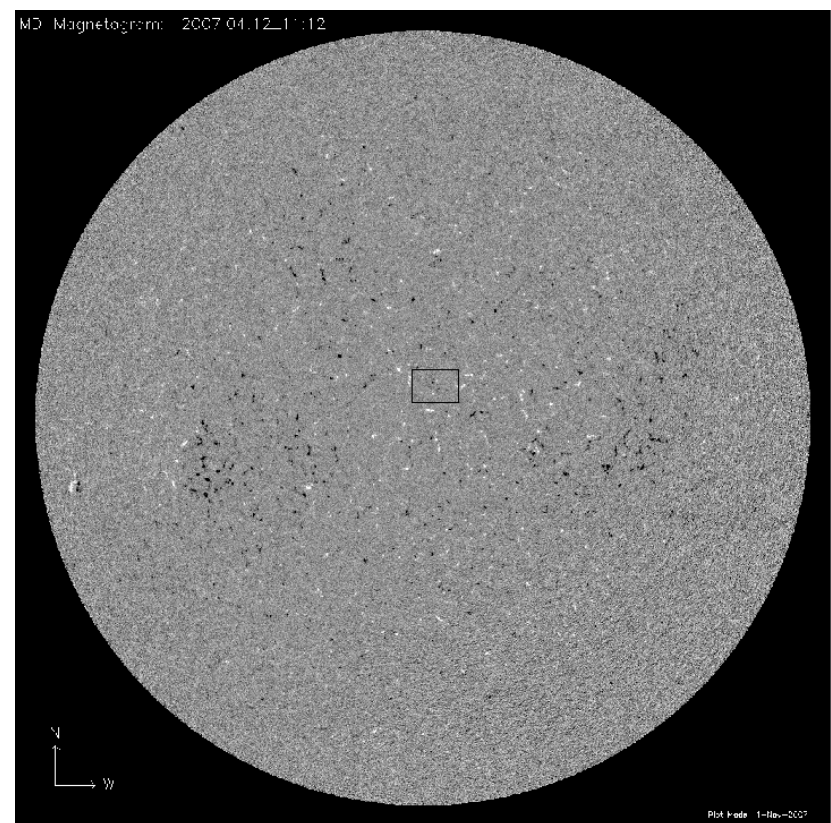

Fig. 1. Magnetic map of the solar disk on April 12, 2007 from MDI showing an extremely low level of solar activity. The $149^{\prime \prime} \times 117^{\prime \prime}$ FOV investigated in this paper is marked.

for those BP that appear embedded in granules, i.e., the above family II.

We avoid these, restricting our selection to small structures, thus excluding extended granular features and, hence, nonmagnetic local $G$-band enhancements embedded. Among the small-scale inter-granular structures (IgS), we remove those that populate the non magnetic branch in the $G$-band-to-continuum scatterplot. The remaining IgS are than sampled by a significant Ca II excess.

The powerful pattern recognition algorithm, followed by a selective sampling of small structures, elimination of nonmagnetic ones by their $G$-band-to-continuum ratio, and classification of magnetic ones by the Ca II excess, qualifies our study as an alternate way to investigate small-scale solar kiloGauss magnetic flux-concentrations at the highest possible spatial resolution and independent of interpreting polarimetric measurements.

\section{Segmentation of G-band features}

We analyzed a set of high-resolution images, observed at an exceptionally deep solar activity minimum on April 12, 2007 (cf., MDI-magnetogram Fig. 1). The Dutch Open Solar Telescope (DOT) was pointed at the intersection of the central meridian with the solar equator $\left(0^{\circ} \mathrm{E} / \mathrm{W} ; 6^{\circ} \mathrm{N}\right)$ by $\mathrm{P}$. Sütterlin, who took image bursts simultaneously in the $G$-band, $\mathrm{Ca}$ II H, $\mathrm{H} \alpha$, blue and red continuum, which he subjected to image reconstruction following de Boer (1996) and finally composed to a 4-image mosaic of $149^{\prime \prime} \times 117^{\prime \prime}$ total field-of-view. That large FOV, marked in Fig. 1 and shown in Fig. 2 as an $\mathrm{H} \alpha$ image, covers $1 / 221$ of the total solar surface, allowing a statistically significant investigation of small-scale magnetic flux-concentrations.

As a first step, the $G$-band image was analyzed using the MLT_4 recognition code $^{1}$ (Bovelet \& Wiehr 2007) to obtain an appropriate pattern of solar structures and their individual sizes in pixel counts. The latter depend to some extent on the

\footnotetext{
1 http://www.gwdg.de/ bbovele; login as "user", password "mlt4".
}

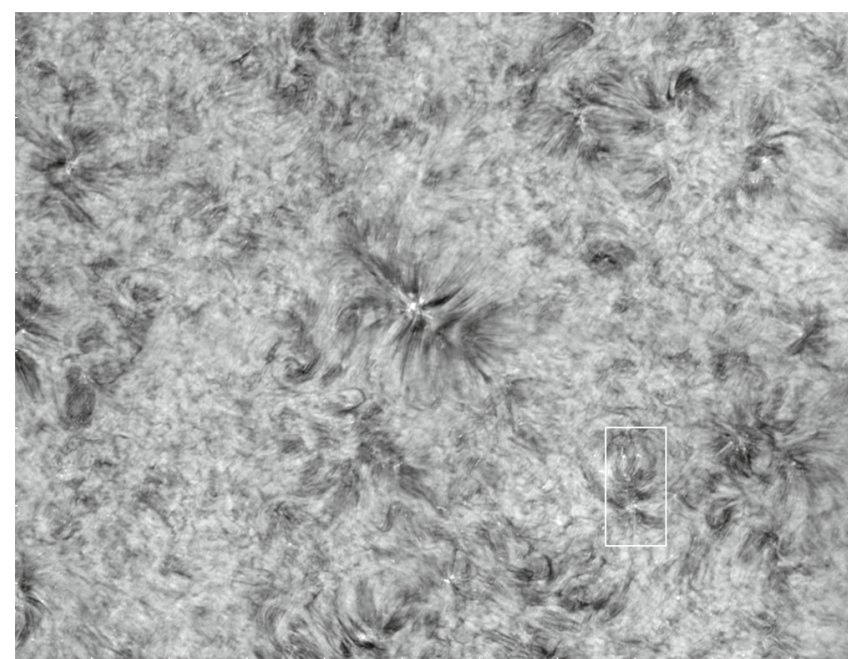

Fig. 2. Reconstructed H $\alpha$ image of a $149^{\prime \prime} \times 117^{\prime \prime}$ region centered on the central meridian and the equator $\left(6^{\circ} \mathrm{N}\right)$ on April 12, 2007, (blue and red wing images added; courtesy P. Sütterlin, Utrecht). The square marks a subregion used for a visualization of the data processing in Fig. 5.

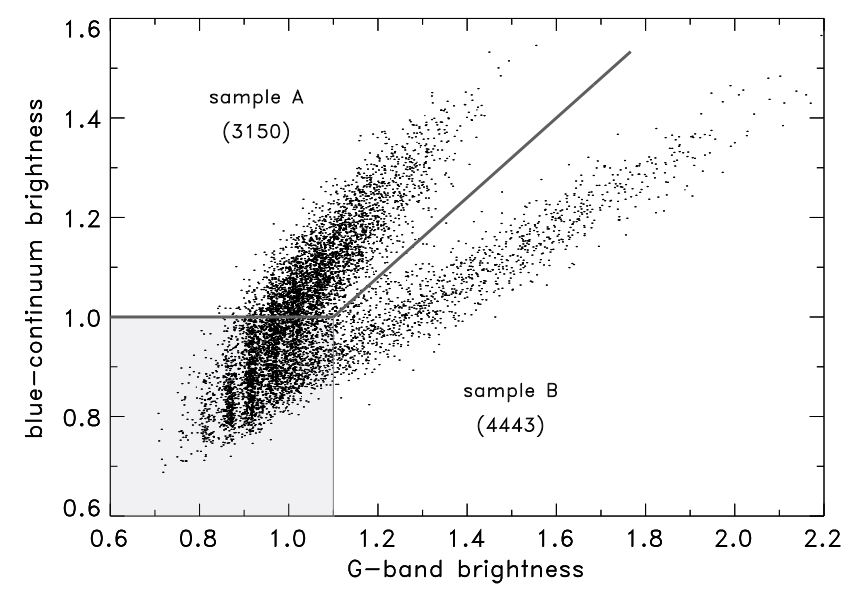

Fig. 3. Scatterplot of blue-continuum and $G$-band brightness for all $7593 \mathrm{IgS}$ selected, grouping in a magnetic (lower) and a non-magnetic branch that are separated by the full line; overlap regime shaded.

"unitary cut level" equally applied to each of the segmented cells: the higher the cut level, the smaller the size. Our final choice of a unitary cut level of 0.5 was adapted to a realistic representation of IgS sizes, accepting slightly broadened intergranular lanes. As a second step, we selected inter-granular structures ("IgS") with an area $\leq 75$ pixels $\left(2 \times 10^{4} \mathrm{~cm}^{2}\right)$. This procedure excludes larger features with local $G$-band enhancements occasionally embedded that are known to be non magnetic (Berger \& Title 2001; Langhans et al. 2002). The suitability of the achieved representation is demonstrated in Fig. 5 for a subfield indicated in Fig. 2.

\section{Selection of IgS with $\mathrm{Ca}$ II $\mathrm{H}$ excess}

For the thus selected total of $7593 \mathrm{IgS}$, we plot the $G$-band excess over the intensity in the neighboring spectral continuum following Berger et al. (1995). The spatial correlation between the two simultaneously observed $G$-band and (blue) continuum images is optimized by a cross-correlation to one pixel $\left(0.071^{\prime \prime}\right)$. Figure 3 shows a scatterplot of the $33 \%$ highest intensity pixels of each $\mathrm{IgS}$ in the continuum and in the $G$-band. The $33 \%$ restriction (rather than its mean value) ensures that the deduced IgS 


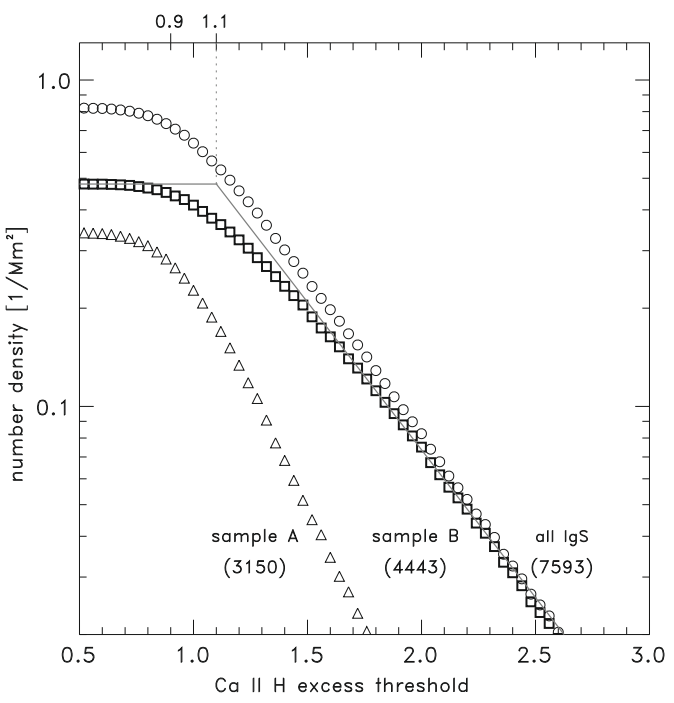

Fig. 4. Number density (logarithmic) as a function of the intrinsic Ca II H excess for all 7593 inter-granular structures, the 3150 above and the 4443 below the division line in Figure 3; flat top and steep decrease approximated by lines intersecting at $E x C a=1.1$.

brightness is largely independent of individual shapes. Figure 3 gives both brightness values in units of the mean photosphere at FOV locations free of local enhancements (e.g., the lower-left region in Fig. 2).

The scatterplot in Fig. 3 shows two branches that, according to measurements by Berger et al. (1995) and to calculations by Shelyag et al. (2004), represent magnetic and non-magnetic IgS and overlap for $G$-band brightness $I^{\mathrm{Gb}}<1.1 \times I_{\text {mean }}^{\mathrm{Gb}}$ (shaded in Fig. 3). Since we do not, a priori, know which of the segmented features are magnetic, we remove, as a third step, 3150 nonmagnetic $\operatorname{IgS}$ in the upper part of the non magnetic branch (sample A), located above the kinked line line inserted in Fig. 3.

Among the remaining $4443 \mathrm{IgS}$ (sample B), we disentangle non-magnetic and magnetic ones by their $\mathrm{Ca}$ II $\mathrm{H}$ excess brightness, assuming magnetic IgS to exhibit a substantial $\mathrm{Ca} I \mathrm{IH}$ excess, whereas non-magnetic IgS are considered to have no significant Ca II H brightening (Lites et al. 1999; Rezaei et al. 2007). We correspondingly remove, as a fourth step, the non magnetic IgS from sample B applying a threshold of the intrinsic $\mathrm{Ca}$ II $\mathrm{H}$ excess defined as

$E x C a:=I_{\text {up }}{ }^{\left(I_{\text {up }} / I_{\text {low }}\right)}$,

with $I_{\text {up }}$ denoting the $\mathrm{Ca}$ II $\mathrm{H}$ brightness contrast over the mean for $33 \%$ of the brightest and $I_{\text {low }}$ for $33 \%$ of the darkest pixels of each IgS. Application of the ratio of both contrasts as the exponent to $I_{\text {up }}$ avoids excluding features with a high but flat intrinsic Ca II H brightness distribution, as $G$-band bright points. Such a criterion mainly selects $\mathrm{IgS}$ with a spatially isolated $\mathrm{Ca}$ II H excess inside their corresponding G-band pattern and excludes faint and "fuzzy" Ca II H structures (widely considered to have an acoustic nature).

The influence of this intrinsic $\mathrm{Ca}$ II $\mathrm{H}$ excess criterion on the number density of IgS is demonstrated in Fig. 4. For sample A, the logarithmic number density of IgS already drops at very low ExCa values, since this group is mainly populated with $\mathrm{IgS}$ from the non magnetic branch without intrinsic Ca II H excess. They mark granular fragments or faint and "fuzzy" Ca II H brightenings that are already removed by a low ExCa value, since both basis and exponent of ExCa are small.

For sample B, the logarithmic number density of $\operatorname{IgS}$ remains constant up to $E x C a \approx 0.9$, then drops, and finally linearly
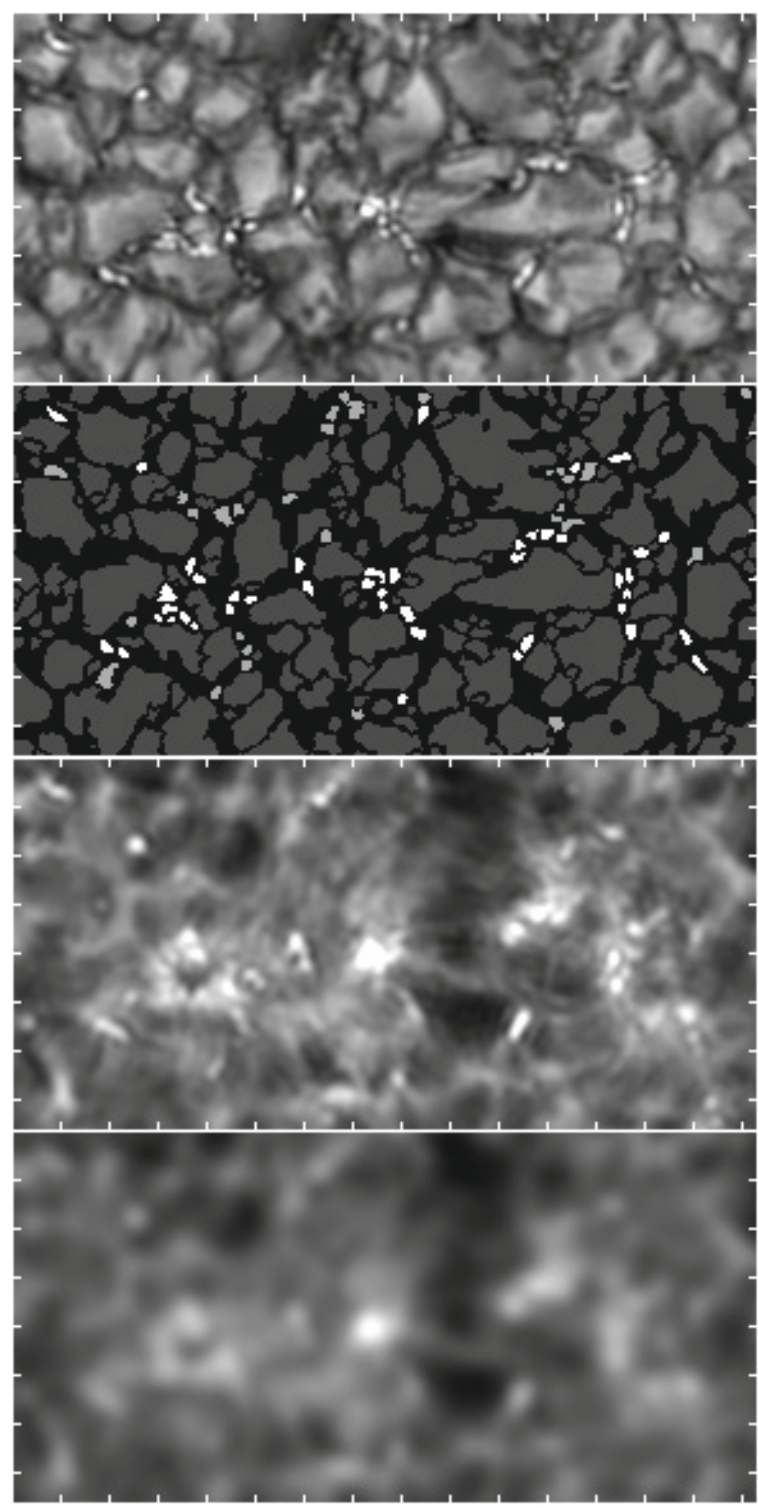

Fig. 5. Sub-region of $10.4^{\prime \prime} \times 20.7^{\prime \prime}$ marked in Fig. 2 and rotated by $-90^{\circ}$ in the light of the $G$-band, together with the corresponding patterns of bright points (BP; white), faint points (FP; light gray), and granules (dark gray); the Ca II H image without and with artificial degradation to $0.75^{\prime \prime}$ spatial resolution; tickmarks: $1 \mathrm{Mm}$.

decreases for $E x C a>1.4$. The high Ca II H excess of IgS in sample B causes already low ExCa thresholds to exclude most of the non-magnetic IgS, then leaving the number density constant. When further increasing the threshold to $E x C a>0.9$, magnetic IgS with $\mathrm{Ca}$ II $\mathrm{H}$ excess are also excluded progressively.

We eventually eliminate from sample B those $\operatorname{IgS}$ that do not reach $E x C a=1.2$, and obtain 2995 remaining $\operatorname{IgS}$, which are $\mathrm{Ca}$ II $\mathrm{H}$ bright and thus reasonably assumed to be magnetic, hence, denoted as "MIgS". Among these, G-band bright MIgS with $I^{\mathrm{Gb}} \geq 1.1 \times I_{\text {mean }}^{\mathrm{Gb}}$ (i.e. beyond the overlap regime) are the known BP. Accordingly, we denote the remaining MIgS with $I^{\mathrm{Gb}}<1.1 \times I_{\text {mean }}^{\mathrm{Gb}}$ as " $G$-band faint points" $(\mathrm{FP})$.

\section{Results}

\subsection{Call brightening}

Figure 5 gives the visual impression that the $\mathrm{CaIIH}$ brightness is not tightly related to the $G$-band brightness. This is quantitatively seen in the scatterplots in Fig. 6. Our 2995 MIgS 

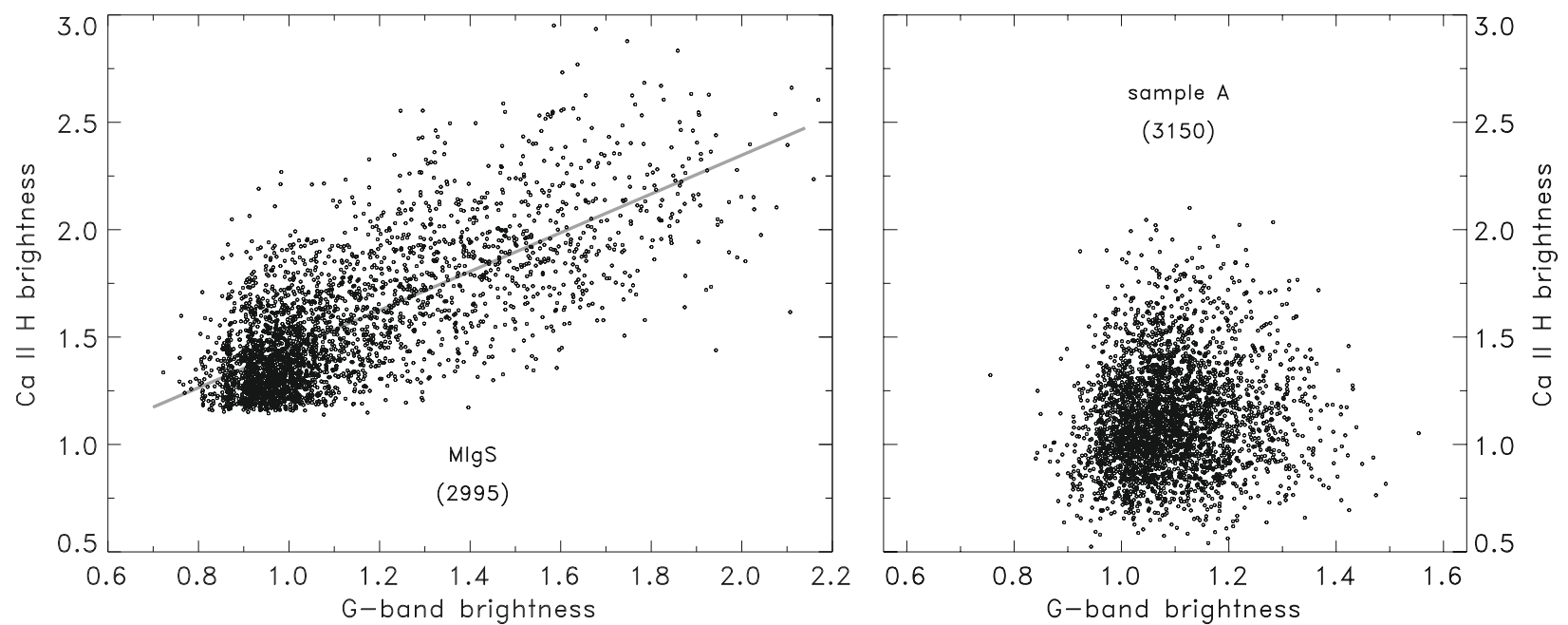

Fig. 6. Scatterplot of the brightness in Ca II H and in the G-band for the $2995 \mathrm{Ca}$ II H bright IgS from sample B (MIgS) and for the $3150 \mathrm{IgS}$ from sample A (right panel), showing a tight relation only for the MIgS (here linearly fitted); the lower limit of the ordinate values marks the influence of the Ca II H threshold applied to sample B.

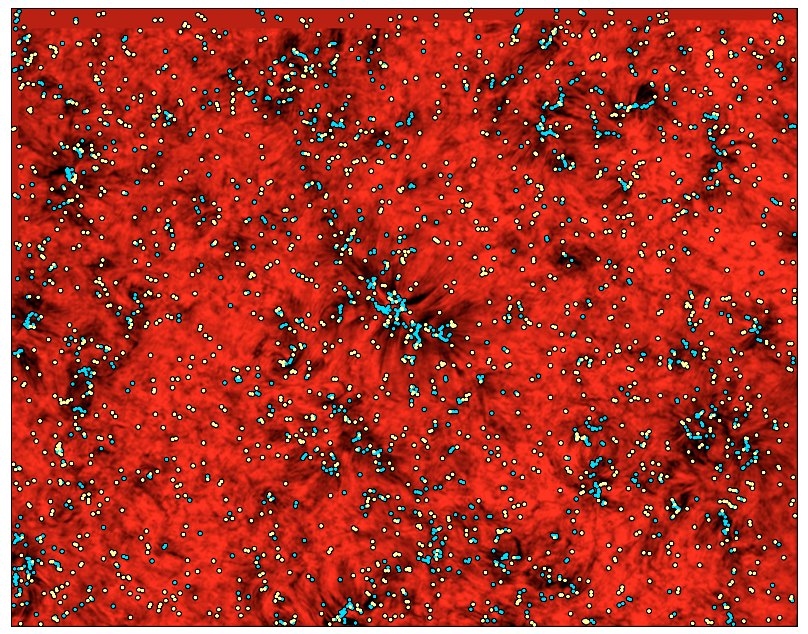

Fig. 7. Location of magnetic inter-granular structures $\operatorname{MIgS}$ within the $\mathrm{H} \alpha$ structures of the $149^{\prime \prime} \times 117^{\prime \prime}$ FOV; white dots mark $G$-band bright MIgS (BP), black dots mark faint ones (FP); the dots represent locations rather than feature sizes; gray peripheral zones not covered by the $\mathrm{H} \alpha$ mosaic; tickmarks: $10 \mathrm{Mm}$. This figure is available in color in electronic form, where white dots are marked blue.

show a fairly good relation that resembles the one found by Rezaei et al. (2007) between the Ca II K index and the magnetic flux density. The smooth transition between BP and FP (at $I^{\mathrm{Gb}}=1.1 \times I_{\text {mean }}^{\mathrm{Gb}}$ ) indicates their similar (magnetic) nature, as found by Bovelet \& Wiehr (2007). The absence of such a relation for the $3150 \mathrm{IgS}$ from sample A (right panel) indicates that these are mainly not (or weak) magnetic.

The findings by Lites et al. (1999) and by Rezaei et al. (2007) that magnetic flux concentrations in the network cell interior do not exhibit a Ca II excess might suggest that most of our MIgS are located at network boundaries. To check this idea, we superpose the $\mathrm{H} \alpha$ image from Fig. 2 with the location of our finally selected MIgS (Fig. 7). This superposition impressively shows that the BP are preferably located near the dark $\mathrm{H} \alpha$ fibrils, which are widely assumed to have their roots in small-scale kilo-Gauss magnetic flux-concentrations.

In contrast, FP appear also outside the $\mathrm{H} \alpha$ fibrils, i.e., in the inter-network (IN). This, however, means that at least part of the
IN flux-concentrations do exhibit marked $\mathrm{Ca}$ II $\mathrm{H}$ excess, since the FP have been equally selected by the ExCa threshold. The discrepancy of this finding to other observations might be due to the higher spatial resolution achieved in our data. This is readily seen in the lowest panel of Fig. 5, which displays the same region as the upper panels but after artificial degradation of the Ca II image simulating a spatial resolution of $0.75^{\prime \prime}(15 \mathrm{~cm}$ telescope aperture). It impressively shows, that even at such moderate degradation, the isolated tiny $\mathrm{Ca}$ II $\mathrm{H}$ bright features are no longer detectable. Our intrinsic Ca II excess criterion even fails to select $\mathrm{IgS}$ for a faint degradation to a $0.5^{\prime \prime}$ spatial resolution. Hence, very high spatial resolution is required to check whether small-scale magnetic flux-concentrations actually exhibit a Ca II excess.

\subsection{Size}

The spatial resolution achieved in our data is proven by the power spectrum (Fig. 8), which drops to twice the noise level at $2 \pi \cdot k \approx 37 \mathrm{Mm}^{-1}$, i.e., $170 \mathrm{~km}$ on the Sun. Accordingly, the histograms of BP and FP in Fig. 9a drop towards small sizes, reaching $170 \mathrm{~km}\left(0.23^{\prime \prime}\right)$ at $5 \%$ of their maxima. The most frequent sizes of the $1152 \mathrm{BP}$ range between 12 and 20 pixels, those of the 1843 FP between 14 and 23. Since our analysis does not need any assumption about feature shapes, we only give diameters for equivalent circular shapes additionally at the upper abscissa of Fig. 9a and obtain most frequent circular diameters of $(220 \pm 30) \mathrm{km}$ for BP and $(250 \pm 30) \mathrm{km}$ for FP.

Towards increasing sizes, the histogram of BP establishes the existence of an upper size limit for the BP (cf., Wiehr et al. 2004; Puschman \& Wiehr 2006; Beck et al. 2007). The corresponding diameter of an equivalent circular shape amounts to $400 \mathrm{~km}$, in agreement with Muller (1985) and with Bovelet \& Wiehr (2005). The FP seem to be systematically larger. We verify this by plotting the $G$-band brightness excess of the MIgS over the neighboring spectral continuum as a function of the size and find that larger MIgS are primarily faint (Fig. 9b).

This is a hint that for larger magnetic flux-concentrations the "hot wall" is wider, thus reducing the brightness excess. In turn, small FP do exist that are of similar size as the BP (lower left part of the data cloud in Fig. 9b); their "closer hot walls" must 


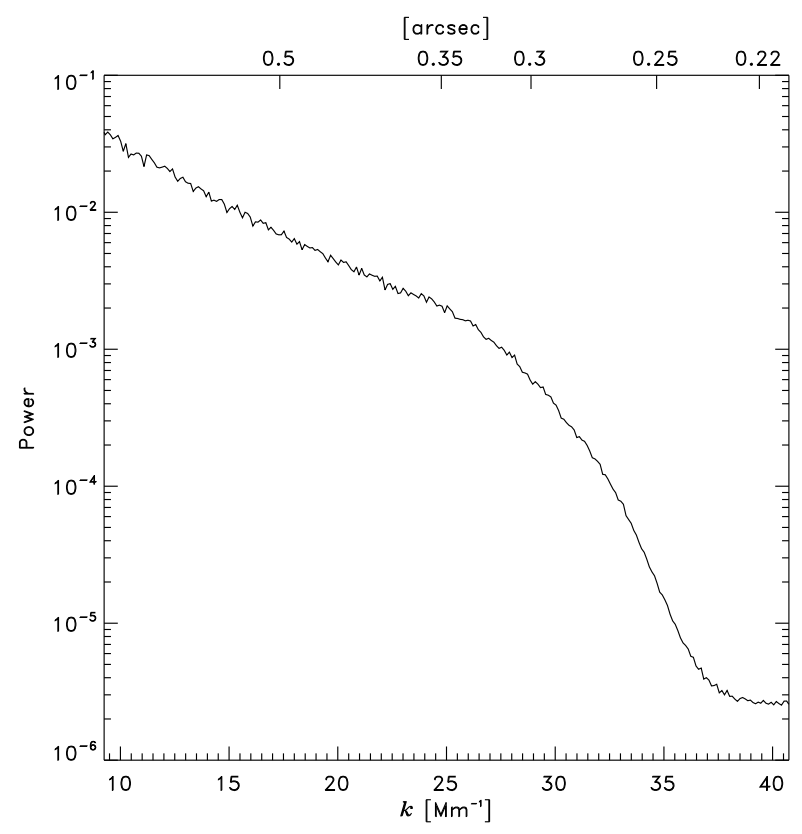

Fig. 8. Power spectrum of the $G$-band image showing a limiting resolution of $2 \pi \times k=37 / \mathrm{Mm}$ of the DOT mosaic image observed and reconstructed by Sütterlin.

Table 1. Characteristics of IgS with $E x C a>1.2$, distinguished for BP $\left(I^{\mathrm{Gb}} \geq 1.1 \times I_{\text {mean }}^{\mathrm{Gb}}\right), \mathrm{FP}\left(I^{\mathrm{Gb}}<1.1 \times I_{\text {mean }}^{\mathrm{Gb}}\right)$, and $\mathrm{MIgS}(\mathrm{BP}$ plus FP) in the entire FOV; MIgS also in regions without pronounced $\mathrm{H} \alpha$ fibrils.

\begin{tabular}{ccccc}
\hline \hline $\begin{array}{c}\text { Sampled } \\
\text { entity }\end{array}$ & $\begin{array}{c}\text { Total } \\
\text { No. }\end{array}$ & $\begin{array}{c}\text { No. dens. } \\
{\left[\mathrm{Mm}^{-2}\right]}\end{array}$ & $\begin{array}{c}\text { Area cover. } \\
{[\%]}\end{array}$ & $\begin{array}{c}\text { Mean flux dens. } \\
{\left[\mathrm{Mx} / \mathrm{cm}^{2}\right]}\end{array}$ \\
\hline BP & 1152 & 0.12 & 0.60 & 9.6 \\
FP & 1843 & 0.20 & 1.40 & 10.7 \\
\hline MIgS in FOV & 2995 & 0.32 & 2.00 & 20.3 \\
MIgS in voids & 888 & 0.22 & 1.52 & 12.8 \\
\hline
\end{tabular}

then be fainter, probably due to a lower magnetic flux density (cf., 4.4). The few data points in the upper right of Fig. 9b represent 14 elongated MIgS (among 2995) that have not been segregated enough by MLT_4; this proves the power of our algorithm.

\subsection{Area contribution}

We reasonably assume that the $G$-band appearance of each MIgS is a realistic measure of the corresponding kilo-Gauss magnetic feature, following Shelyag et al. (2004), who state that the "spatial $G$-band pattern matches the spatial structure of the magnetic feature". Hence, the sum of all 2995 MIgS sizes may be considered as an actual area contribution in the $9.2 \times 10^{19} \mathrm{~cm}^{2}$ FOV $\left(3.48 \times 10^{6} \mathrm{px}\right)$. The $2995 \mathrm{MIgS}$ cover $1.85 \times 10^{18} \mathrm{~cm}^{2}$ (69719 px) corresponding to $2.0 \%$ of the FOV and a number density of $0.32 \mathrm{Mm}^{-2}$ (Table 1), i.e., the same as found by Sánchez Almeida et al. (2004).

The location of BP and FP in the $\mathrm{H} \alpha$ image (Fig. 7) allows to distinguish their affiliation to fibril and non-fibril (IN) regions. The "voids of fibrils" appear to be homogeneously populated by $\mathrm{FP}$, whereas the $\mathrm{BP}$ primarily occur in centers of fibrils regions, as is expected from their nature as magnetic footpoints. For a quantitative analysis, we approximated those complementary regions by a binary mask, obtained from sufficient smoothing of the $\mathrm{H} \alpha$ image. We find a number density of $0.22 \mathrm{Mm}^{-2}$ in the fibril voids, i.e., about $2 / 3$ of the $0.32 \mathrm{Mm}^{-2}$ found for the entire
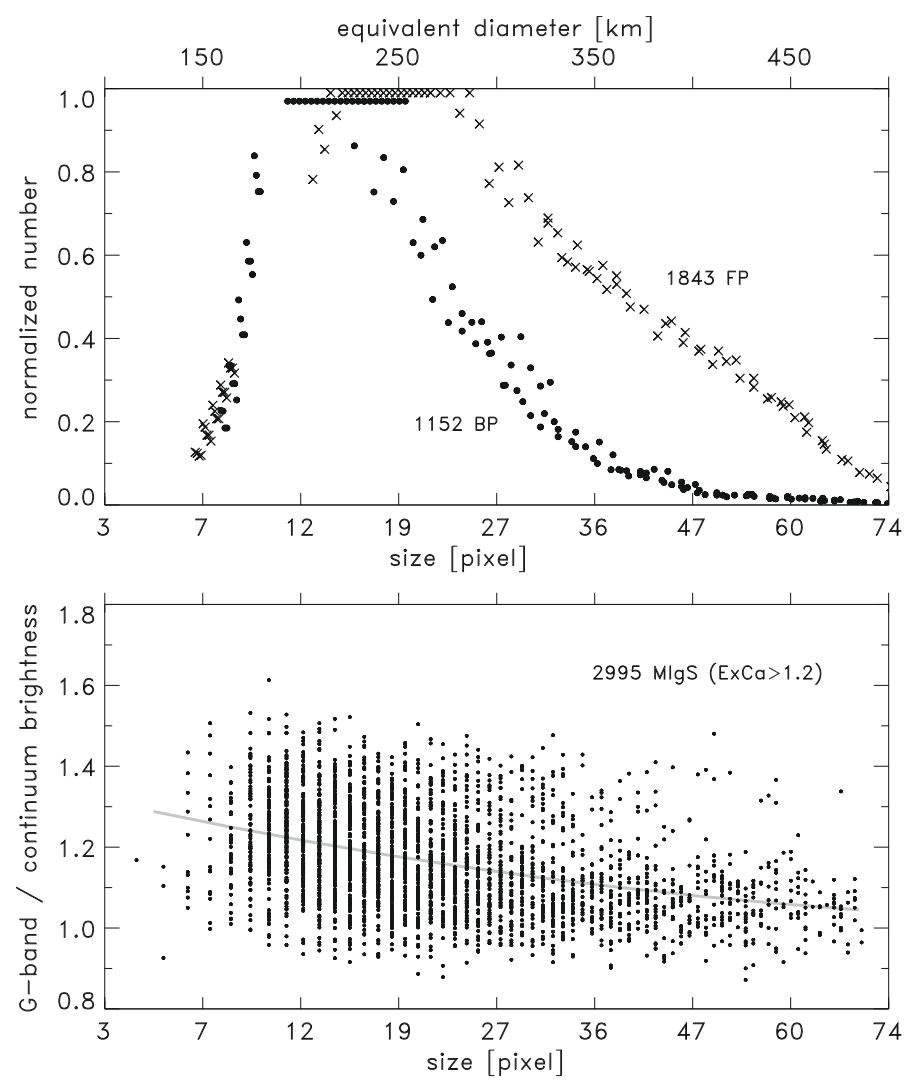

Fig. 9. Size histograms of $1152 \mathrm{BP}$ and $1843 \mathrm{FP}$ from sample B. The lower abscissa gives realistic sizes in pixel counts; the upper abscissa gives equivalent diameters of corresponding circular shapes. The data points represent counts per bin-size of 21 maximum-scaled histograms with different bin-sizes that are overplotted to provide enough invariance from subjective sampling. The lower panel shows the $G$-band brightness excess (with respect to the neighboring spectral continuum) as a function of the size for all $2995 \mathrm{MIgS}$ (BP plus FP).

FOV (Table 1). We verified that this value does not significantly depend on the smoothing for a binary mask.

\subsection{Estimate of magnetic parameters}

To obtain the mean magnetic flux density of the MIgS in the FOV, we might multiply their area contribution of $2 \%$ by a "typical" value for an intrinsic flux density of $1 \mathrm{kG}$ and obtain a mean (unsigned) flux density of $20 \mathrm{G}$. A possible way estimating the intrinsic flux of each MIgS may be obtained from the relation between the $G$-band brightness and the magnetic flux density, as modeled by Shelyag et al. (2004). There, in Fig. 4, most of the scatter of the $G$-band brightness arises from the spatially varying continuum level in their calculation area (also containing granules). Our sample of MIgS, however, is restricted to small intergranular features with a certain $G$-band excess and will be less influenced by continuum variations. We may thus consider the mean MHD relation as calibration.

The modeled relation refers to each pixel of a magnetic feature. We averaged the $G$-band brightness of the pixels of each MIgS and used that mean for calibration (left panel of Fig. 10), thus obtaining the intrinsic magnetic flux density. Multiplying this value with the corresponding area (returned by MLT_4) yields a flux value for each MIgS. The middle panel of Fig. 10 shows these flux values as a function of the mean $G$-band brightness for each of our 2995 MIgS. 

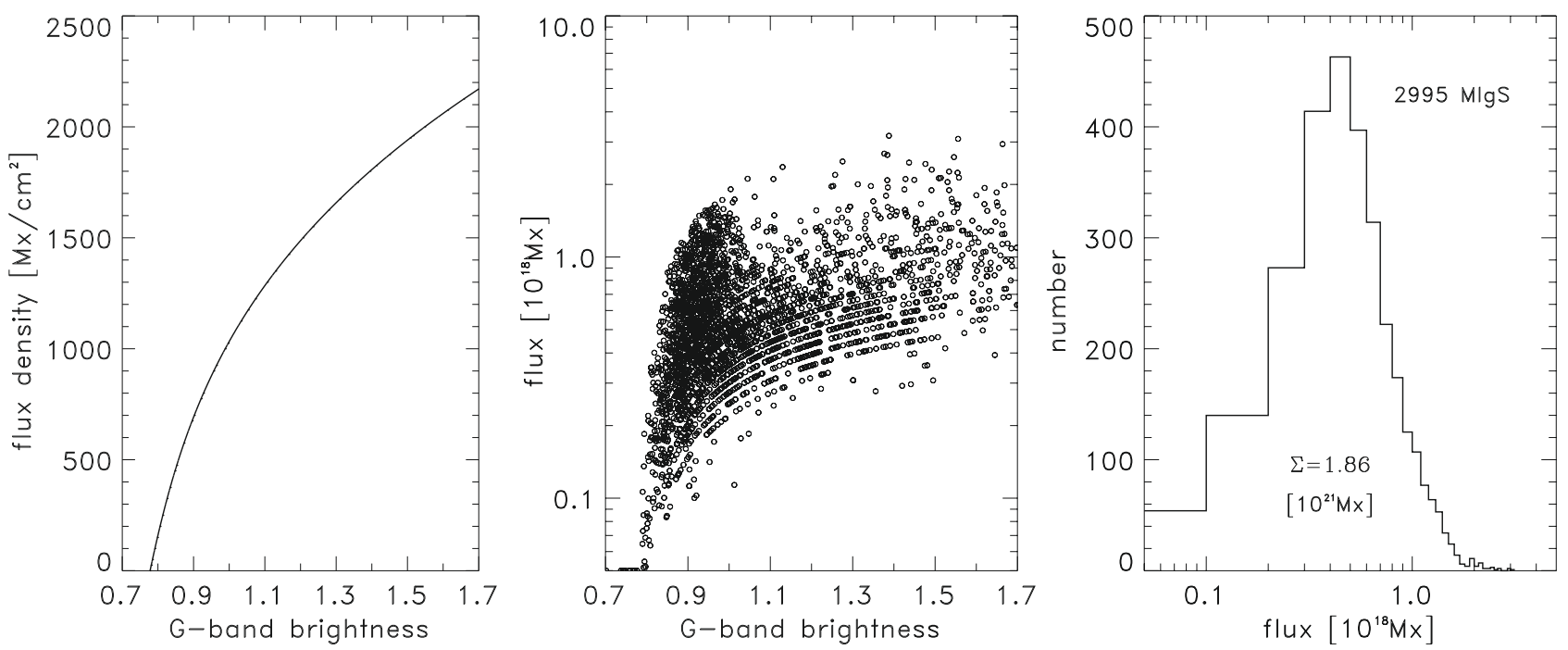

Fig. 10. Calibration curve for MIgS (left) averaged from Fig. 4 in Shelyag et al. (2004); scatterplot of $G$-band brightness and magnetic flux (middle) and flux histogram (right panel) of 2995 MIgS.

The flux values depend to some extent on the unitary cut level (0.5; cf., Sect. 2) applied to each segmented cell after normalization to its brightness maximum in phase 4 of MLT_4 (cf., Bovelet \& Wiehr 2007): the higher the cut level, the smaller the size. Choosing a lower cut threshold would expand MIgS to their peripheral pixels that are fainter and thus would lower the mean $G$-band brightness of the features. The calibration curve (left panel of Fig. 10) will then assign lower flux densities, and the flux of this expanded MIgS will increase by only a few percent. The influence of our unitary cut level on the deduced MIgS flux is thus negligible.

\subsection{Mean flux in the FOV}

The histogram of the flux of the MIgS (right panel of Fig. 10) shows a narrow distribution with most frequent values of $0.45 \times 10^{18} \mathrm{Mx}$ and a total flux of $1.86 \times 10^{21} \mathrm{Mx}$ for all $2995 \mathrm{MIgS}$. If we divide the latter value by the total area of the $2995 \mathrm{MIgS}\left(1.85 \times 10^{18} \mathrm{~cm}^{2}\right)$, we obtain a most frequent MIgS flux density of $1005 \mathrm{Mx} / \mathrm{cm}^{2}$, showing that the kilo-Gauss assumption is a realistic mean over the entire FOV. But the most frequent flux density largely differs between BP and FP, amounting to $1597 \mathrm{Mx} / \mathrm{cm}^{2}$ for the BP and to $775 \mathrm{Mx} / \mathrm{cm}^{2}$ for the FP.

The difference between BP and FP is not that great, however, for the mean flux density in the entire FOV. Dividing the total flux of the $1152 \mathrm{BP}$ and that of the $1842 \mathrm{FP}$ separately by the entire FOV area, we obtain $9.6 \mathrm{Mx} / \mathrm{cm}^{2}$ for the BP and $10.6 \mathrm{Mx} / \mathrm{cm}^{2}$ for the FP (which are more frequent but less magnetic, see above). The sum of both values gives a mean flux density over the FOV of $20.3 \mathrm{Mx} / \mathrm{cm}^{2}$ for all $2995 \mathrm{MIgS}$, a number that ranges between that by Daras-Papamargaritis \& Koutchmy $\left(1983 ; 10^{21} \mathrm{Mx} / 10^{20} \mathrm{~cm}^{2}\right)$ and the one by Sánchez Almeida $\left(2003 ; 30 \mathrm{Mx} / \mathrm{cm}^{2}\right.$ for his full FOV).

In the "fibril voids" seen in Fig. 2, we find $0.22 \mathrm{MIgS} / \mathrm{Mm}^{2}$ covering $1.52 \%$ of the corresponding void area (Table 1). Assuming kilo-Gauss field strengths for each MIgS, we obtain a mean flux density in the fibril voids of $15.2 \mathrm{Mx} / \mathrm{cm}^{2}$, in perfect agreement with the finding by Sánchez Almeida (2003; for the network interior). However, attributing to each MIgS its individual magnetic flux via the calibration curve (left panel of Fig. 10) yields a mean flux density of only $12.8 \mathrm{Mx} / \mathrm{cm}^{2}$ for the void areas. This value is lower than the above one for kilo-Gauss fields, since the fibril voids are primarily populated by FP, which exhibit lower most frequent flux densities. We verified that the reduction of the mean flux density in fibril voids as compared to that in the entire FOV does not significantly depend on the binary mask used to exclude fibril regions (cf., Table 1).

\section{Conclusions}

The segmentation and selection of small-scale inter-granular structures with a significant $\mathrm{Ca}$ II $\mathrm{H}$ excess allows lower limits to be determined for the number density and fractional area coverage of inter-granular kilo-Gauss magnetic flux-concentrations. A magnetic calibration of each feature yields a mean (unsigned) magnetic flux density in a quiet solar region close to existing results. Our method offers an alternate approach to polarimetry, although it inevitably misses those magnetic structures that do not exhibit a $\mathrm{CaIIH}$ excess and "elusive magnetic structures" from fluctuations in thermodynamic properties (which Sánchez Almeida 2000, estimate to carry at least half of the solar magnetic flux).

In case the total flux in our disk center FOV were representative of the entire solar surface $(221 \times$ FOV $)$, the Sun would be covered by $>0.6 \times 10^{6} \mathrm{MIgS}$ with a total flux of $>4.1 \times 10^{23} \mathrm{Mx}$. Considering also magnetic structures which are not covered by our selection, the Sun at activity minimum may well be covered by a few millions of MIgS with a total flux of $10^{24} \mathrm{Mx}$ or even more.

Our method offers a quantitative investigation of highly resolved (reconstructed) intensity images, avoiding the ambiguities of the Stokes calibration procedure and allowing closely neighboring opposite polarities to be disentangled up to the spatial resolution achieved in two-dimensional brightness images.

Acknowledgements. Drs. J. Sánchez Almeida, M. Schüssler, and O. Steiner contributed helpful suggestions. Dr. P. Sütterlin performed the observations and image reconstruction, Dr. J. Hirzberger the power spectra. An anonymous referee pointed out some critical problems. The computational work was performed under PV-WAVE (by Visual Numerics, Inc.). 


\section{References}

Beck, C., Bello Rubio, L. R., Schlichenmaier, R., \& Sütterlin, P. 2007, A\&A, 472, 607

Berger, T. E., Shrijver, C. J, Shine, R. S., Tarbell, T. D., \& Title, A. M. 1995, ApJ, 454, 531

Berger, T. E., Löfdahl, M. G., Shine, R. S., \& Title, A. M. 1998, ApJ, 495, 973

Bovelet, B., \& Wiehr, E. 2001, Sol. Phys., 201, 13

Bovelet, B., \& Wiehr, E. 2003, A\&A, 412, 249

Bovelet, B., \& Wiehr, E. 2007, Sol. Phys., 243, 121

De Wijn, A. G., Rutten, R. J., Haverkamp, E. M. W. P., \& Sütterlin, P. 2005, A\&A, 441, 1183

Domínguez Cerdeña, I., Sánchez Almeida, J., \& Kneer, F., 2006, ApJ, 636, 469

Daras-Papamargaritis, H., \& Koutchmy, S. 1983, A\&A, 125, 280

Langhans, K., Schmidt, W., \& Tritschler, A. 2002, A\&A, 394, 1069

Lites, B. W., Rutten, R. J., \& Berger, T. E. 1999, ApJ, 517, 1013

Lites, B. W., Kubo, M., Socas-Navarro, H., et al. 2008, ApJ, 672, 1237
Muller, R. 1983, Sol. Phys., 85, 113

Muller, R., \& Roudier, T., 1984, Sol. Phys., 94, 33

Puschman, K. G., \& Wiehr, E. 2006, A\&A, 445, 337

Rezaei, R., Schlichenmaier, R., Beck, C. A. R., Bruls, J. H. M. J., \& Schmidt, W. 2007, A\&A, 466, 1131

Sánchez Almeida, J. 2000, ApJ, 544, 1135

Sánchez Almeida, J. 2003, A\&A, 411, 615

Sánchez Almeida, J., Márquez, I., Bonet, J. A., Domínguez Cerdeña, I., \& Muller, R. 2004, ApJ, 609, L-91

Scharmer, G. B., Bjelsko, K., Korhonen, T. K, Lindberg, B., \& Pettersen, B. 2003, Proc. SPIE, 4853, 370

Shelyag, S., Schüssler, M., Solanki, S., Berdyugina, S. V., \& Vögler, A. 2004, A\&A, 427, 335

Title, A. E., \& Berger, T. E. 1996, ApJ, 463, 797

Vögler, A., \& Schüssler, M. 2007, A\&A, 465, L-43

Wiehr, E., Bovelet, B., \& Hirzberger, J. 2004, A\&A, 422, L63 